\title{
A sharp inequality for the variance with respect to the Ewens Sampling Formula
}

\author{
Ž. Baronėnas, E. Manstavičius and P. Šapokaitè
}

March 16, 2020

\begin{abstract}
The variance of a linear statistic defined on the symmetric group endowed with the Ewens probability is examined. Despite the dependence of the summands, it can be bounded from above by a constant multiple of the sum of variances of the summands. We find the exact value of this constant. The analysis of the appearing quadratic forms and eigenvalue search is built upon the exponential matrices and discrete Hahn's polynomials.
\end{abstract}

\section{Introduction and results}

The variance of a linear statistic defined on the symmetric group endowed with the Ewens probability is examined in the paper. The main obstacle to overcome in this seemingly simple problem is the dependence of the summands. We propose an approach built upon exponential matrices and special functions.

Let $\mathbf{S}_{n}$ denote the symmetric group of permutations $\sigma$ acting on $n \in \mathbf{N}$ letters. Each $\sigma \in \mathbf{S}_{n}$ has a unique representation (up to the order) by the product of independent cycles $\kappa_{i}$ :

$$
\sigma=\kappa_{1} \cdots \kappa_{w}
$$

\footnotetext{
${ }^{0} A M S 2000$ subject classification. Primary 60C05; secondary 05A16, $20 \mathrm{P} 05$. Key words and phrases. Random permutation, linear statistics, quadratic form, eigenvalue, discrete Hahn's polynomials, Turán-Kubilius inequality.
} 
where $w=w(\sigma)$ denotes the number of cycles. Denote by $k_{j}(\sigma) \geq 0$ the number of cycles in (11) of length $j$ for $1 \leq j \leq n$ and introduce the cycle vector $\bar{k}(\sigma)=\left(k_{1}(\sigma), \ldots, k_{n}(\sigma)\right)$.

As usual, set $(x)_{m}=x(x+1) \cdots(x+m-1), m \in \mathbf{N}_{0}:=\mathbf{N} \cup\{0\}$, for the increasing factorial. Denote also

$$
\Theta(m)=(\theta)_{m} / m !=\left[x^{m}\right](1-x)^{-\theta},
$$

where $\left[x^{m}\right] f(x)$ stands for the $m$ th coefficient of a power series $f(x)$ and $\theta>0$ is a parameter. The Ewens Probability Measure $\nu_{n, \theta}$ on $\mathbf{S}_{n}$ is defined by

$$
\nu_{n, \theta}(\{\sigma\})=\theta^{w(\sigma)} /(\theta)_{n}, \quad \sigma \in \mathbf{S}_{n}
$$

Set $\ell(\bar{s})=1 s_{1}+\cdots+n s_{n}$ for a vector $\bar{s}=\left(s_{1}, \ldots, s_{n}\right) \in \mathbf{N}_{0}{ }^{n}$. Equality $\ell(\bar{k}(\sigma))=n$, valid for each $\sigma \in \mathbf{S}_{n}$, shows the dependence of the r.vs $k_{j}(\sigma)$ with respect to $\nu_{n, \theta}$. Throughout the paper, following the tradition of the probabilistic number theory, we leave out the elementary event $\sigma$ in all r.vs defined on the probability space $\left(\mathbf{S}_{n}, 2^{\mathbf{S}_{n}}, \nu_{n, \theta}\right)$. It is well known (see, for example, [1, Sect. 2.3]) that the distribution of $\bar{k}(\sigma)$ can be written as the conditional distribution of $\bar{\xi}=\left(\xi_{1}, \ldots, \xi_{n}\right)$, where $\xi_{j}, 1 \leq j \leq n$, are mutually independent Poisson r.vs given on some probability space $\{\Omega, \mathcal{F}, \mathbf{P}\}$ with parameter $\mathbf{E} \xi_{j}=\theta / j$. Indeed,

$$
\nu_{n, \theta}(\bar{k}(\sigma)=\bar{s})=\mathbf{1}\{\ell(\bar{s})=n\} \Theta(n)^{-1} \prod_{j=1}^{n}\left(\frac{\theta}{j}\right)^{s_{j}} \frac{1}{s_{j} !}=\mathbf{P}(\bar{\xi}=\bar{s} \mid \ell(\bar{\xi})=n) .
$$

Here $\mathbf{1}\{\cdot\}$ stands for the indicator function. The probability in (2), ascribed to the vector $\bar{s} \in \mathbf{N}_{0}^{n}$, is called the Ewens Sampling Formula. It has been introduced by W. J. Ewens [5] to model the mutation of genes. For a comprehensive account of the recent applications of this ubiquitous distribution in combinatorics and statistics, see [1], [7], [6], or survey [4] and the subsequent comments on it.

We prefer to stay within the theory of random permutations. Apart from $w(\sigma)$, other linear statistics (or completely additive functions)

$$
h(\sigma):=a_{1} k_{1}(\sigma)+\cdots+a_{n} k_{n}(\sigma)
$$

where $\bar{a}:=\left(a_{1}, \ldots, a_{n}\right) \in \mathbf{R}^{n}$ is a non-zero vector, continue to raise an interest. For example, $h(\sigma)$ with $a_{j}=\log j, j \leq n$, is a good approximation 
for the logarithm of the group-theoretical order of $\sigma \in \mathbf{S}_{n}$ (see [1] or [20]). The case with $a_{j}=\{x j\}$, where $\{u\}$ stands for the fractional part of $u \in \mathbf{R}$, is met in the theory of random permutation matrices (see [19]).

For an arbitrary $h(\sigma)$, the problem of finding necessary and sufficient conditions, assuring the weak convergence of distributions

$$
\nu_{n, \theta}(h(\sigma)-\alpha(n) \leq x \beta(n)),
$$

where $\alpha(n) \in \mathbf{R}$ and $\beta(n) \rightarrow \infty$ as $n \rightarrow \infty$, is still open (see [1, Sect. 8.5] or [12] and the references therein). Obstacles in the necessity part arise because of the dependence of the summands as shown by (21). This also happens in the analysis of power moments carried out by the second author [10] and [11] even in the case $\theta=1$. Let us now focus on the variance.

By $\mathbf{E}_{n, \theta} f(\sigma)$ and $\mathbf{V} a r_{n, \theta} f(\sigma)$ we denote the mean value and the variance of a r.v. $f(\sigma)$ defined on $\mathbf{S}_{n}$ with respect to $\nu_{n, \theta}$. For the particular function $h(\sigma)$ in (3), we also set $A_{n, \theta}(\bar{a})=\mathbf{E}_{n, \theta} h(\sigma)$ and $D_{n, \theta}(\bar{a})=\mathbf{V}_{a r_{n, \theta}} h(\sigma)$. Applying Watterson's [18] formulas (see [1, (5.6), p. 96]) for the factorial moments of $k_{j}(\sigma)$, one easily finds (see [13] for the details) the expressions

$$
A_{n, \theta}(\bar{a})=\theta \sum_{j \leq n} \frac{a_{j}}{j} \frac{\Theta(n-j)}{\Theta(n)}
$$

and

$$
\begin{aligned}
D_{n}(\bar{a})= & \theta \sum_{j \leq n} \frac{a_{j}^{2}}{j} \frac{\Theta(n-j)}{\Theta(n)}+\theta^{2} \sum_{i+j \leq n} \frac{a_{i} a_{j}}{i j} \frac{\Theta(n-i-j)}{\Theta(n)} \\
& -\theta^{2}\left(\sum_{j \leq n} \frac{a_{j}}{j} \frac{\Theta(n-j)}{\Theta(n)}\right)^{2} \\
= & : \theta B_{n}(\bar{a})+\theta^{2} \Delta_{n}(\bar{a}),
\end{aligned}
$$

if $n \geq 2$ and

$$
B_{n}(\bar{a})=\sum_{j \leq n} \frac{a_{j}^{2}}{j} \frac{\Theta(n-j)}{\Theta(n)} .
$$

The latter quantity is close to the sum of variances of the summands in the definition of $h(\sigma)$. In fact, formula (4) from [13] shows that

$$
\sum_{j \leq n} a_{j}^{2} \mathbf{V} a r_{n, \theta} k_{j}(\sigma)-\theta B_{n}(\bar{a})=O\left(n^{-(1 \wedge \theta)} B_{n}(\bar{a})\right),
$$


where $a \wedge b=\min \{a, b\}$ if $a, b \in \mathbf{R}$, with an absolute constant in the symbol $O(\cdot)$. We also have (see [13])

$$
D_{n}(\bar{a}) \leq C \theta B_{n}(\bar{a})
$$

uniformly in $n \geq 2$ with an absolute constant $C$ which can be specified. If $\theta \geq 1$, one can take $C=2$. The purpose of the present paper is to find the exact value of $C$ in (6).

Theorem 1. Let $\theta>0$ be arbitrary and $n \geq 2$. Then

$$
\tau_{n}(\theta):=\sup \left\{\frac{D_{n}(\bar{a})}{\theta B_{n}(\bar{a})}: \quad \bar{a} \in \mathbf{R}^{n} \backslash\{0\}\right\}=\frac{\theta+2}{\theta+1} .
$$

The supremum is achieved taking $a_{j}=(\theta+2) j^{2}-(2 n+\theta) j$ where $1 \leq j \leq n$.

The pioneering results obtained in [10] and [14] showed that $\tau_{n}(1)=$ $3 / 2+O\left(n^{-1}\right)$ and $\tau_{n}(2)=4 / 3+O\left(n^{-1}\right)$. The approach originated in Kubilius' paper [9] was based upon the extremal properties of the Jacobi polynomials. It unavoidably added a vanishing error term to the result. Recently J. Klimavičius and the second author [8] established that $\tau_{n}(1)=3 / 2$ for all $n \geq 2$. Theorem 1 resumes the research for an arbitrary $\theta>0$. It is directly related to the above mentioned problem concerning distributions (4). Applying Theorem 1, we obtain that the weak convergence of (4) with $\beta(n)=\sqrt{\theta B_{n}(a)}$, which is natural to use, can take place only to the limit laws having variance not exceeding $(\theta+2) /(\theta+1)$.

By virtue of (2), the result can be reformulated for the conditional variance of the linear statistics

$$
Y_{n}:=a_{1} \xi_{1}+\cdots+a_{n} \xi_{n}
$$

We obtain the following optimal inequality.

Corollary. Let $n \geq 2$ and $a_{j} \in \mathbf{R}, 1 \leq j \leq n$, be arbitrary. Then

$$
\operatorname{Var}\left(Y_{n} \mid \ell(\bar{\xi})=n\right) \leq \frac{\theta(\theta+2)}{\theta+1} \sum_{j=1}^{n} \frac{a_{j}^{2}}{j} \frac{\Theta(n-j)}{\Theta(n)}
$$

The problem concerns the quadratic forms $\Delta_{n}(\bar{a})$ and $B_{n}(\bar{a})$. The substitution

$$
a_{j}=\left(\frac{j \Theta(n)}{\Theta(n-j)}\right)^{1 / 2} x_{j}, \quad 1 \leq j \leq n
$$


reduces $B_{n}(\bar{a})$ to the square of Euclidean norm $\|\bar{x}\|^{2}$ of the vector $\bar{x}=$ $\left(x_{1}, \ldots, x_{n}\right) \in \mathbf{R}^{n}$. Then $\Delta_{n}(\bar{a})$ becomes a quadratic form, denoted afterwards by $\mathcal{M}_{n}(\bar{x}):=\bar{x} M_{n} \bar{x}^{\prime}$, where $\bar{x}^{\prime}$ is the column-vector and $M_{n}=\left(\left(m_{i j}\right)\right)$, $1 \leq i, j \leq n$, is the matrix with entries

$$
m_{i j}=\frac{\Theta(n-i-j)}{(i j \Theta(n-i) \Theta(n-j))^{1 / 2}}-\left(\frac{\Theta(n-i)}{i \Theta(n)}\right)^{1 / 2}\left(\frac{\Theta(n-j)}{j \Theta(n)}\right)^{1 / 2} .
$$

Here we assume that $\Theta(-k)=0$ if $k \in \mathbf{N}$. Now, by virtue of (15),

$$
\begin{aligned}
\tau_{n, \theta} & =1+\theta \sup _{\substack{x \neq \overline{0} \\
x \neq}}\left(\|\bar{x}\|^{-2} \mathcal{M}_{n}(\bar{x})\right)=1+\theta \sup _{\bar{x} \neq \overline{0}}\left(\|\bar{x}\|^{-2} \sum_{r=1}^{n} \mu_{r} x_{r}^{2}\right) \\
& =1+\theta \max _{1 \leq r \leq n} \mu_{r},
\end{aligned}
$$

where $\left\{\mu_{1}, \ldots, \mu_{n}\right\}$ is the spectrum of matrix $M_{n}$. So, Theorem 1 follows from the following proposition.

Theorem 2. The spectrum of the matrix $M_{n}$ comprises

$$
\mu_{r}=\frac{(-1)^{r}(r-1) !}{(\theta)_{r}}, \quad 1 \leq r \leq n .
$$

For the eigenvector corresponding to the maximal $\mu_{2}$, one may take the vector with coordinates

$$
((\theta+2) j-(2 n+\theta))(j \Theta(n-j))^{1 / 2}, \quad 1 \leq j \leq n .
$$

The proof of Theorem 2 presented in the next section is built upon exponential matrices.

The problem of finding the remaining eigenvectors of matrix $M_{n}$ also raises an interest. We solve it basing upon particular cases of the generalized hypergeometric series which are exposed, for example, in [3, Chap. 5]. The hint to exploit them stems from [8]. Let us confine ourselves to the case of polynomials which, in the traditional notation, can be written as

$$
{ }_{p+2} F_{q}\left(-m,-x,\left(a_{p}\right) ;\left(b_{q}\right) ; z\right)=\sum_{k=0}^{m} \frac{(-m)_{k}(-x)_{k}\left(a_{1}\right)_{k} \cdots\left(a_{p}\right)_{k}}{\left(b_{1}\right)_{k} \cdots\left(b_{q}\right)_{k} k !} z^{k},
$$

where $p, q, m \in \mathbf{N}_{0}, a_{1}, \ldots, a_{p} ; b_{1}, \ldots, b_{q} \in \mathbf{R}$ are parameters. Moreover, it suffices to reckon the discrete Hahn's polynomials

$$
Q_{r}(x ; \alpha, \beta, n)={ }_{3} F_{2}(-r,-x, r+\alpha+\beta+1 ; \alpha+1,-n+1 ; 1)
$$


by specifying the parameters to $\alpha=1$ and $\beta=\theta-1$. In this case,

$$
q_{r}(x)=Q_{r}(x-1 ; 1, \theta-1, n), \quad 0 \leq r \leq n-1,
$$

have the following orthogonality property:

$$
<q_{l}, q_{r}>:=\sum_{j=1}^{n} j q_{l}(j) q_{r}(j) \Theta(n-j)=\delta_{l r} \pi_{r}^{2},
$$

where $\delta_{l r}$ is the Kronecker symbol and $\pi_{r}>0$. Note that, up to a constant factor, $q_{r}(x), 1 \leq r \leq n-1$, can be obtained uniquely by the Gram-Schmidt orthogonalization procedure starting with $q_{0}(x)=1$ and form an orthogonal basis in the vector space of polynomials whose degrees do not exceed $n-1$. Exploiting this, in the isomorphic Euclidean space $\mathbf{R}^{n}$, we easily find the needed canonical basis for the matrix $M_{n}$.

Theorem 3. The system of the vectors

$$
\bar{e}_{r}=\left(e_{r 1}, \ldots, e_{r n}\right), \quad 1 \leq r \leq n,
$$

where

$$
e_{r j}=\pi_{r-1}^{-1} q_{r-1}(j) \sqrt{j \Theta(n-j)}, \quad 1 \leq j \leq n,
$$

is an orthonormal basis in $\mathbf{R}^{n}$. Moreover, the vector $\bar{e}_{r}$ is the eigenvector of matrix $M_{n}$ corresponding to $\mu_{r}$ for each $1 \leq r \leq n$.

The proof will be presented in the last section of the paper.

Finally, the distributions of mappings defined on random permutations taken according to the Ewens probability are close to that defined on logarithmic decomposable combinatorial structures (see [1]); therefore, we hope that our method is applicable when estimating the variances of similar statistics defined in such classes.

\section{Proof of Theorem 2}

The idea is to find a matrix $L_{n}$ such that the product

$$
\mathrm{e}^{L_{n}} M_{n} \mathrm{e}^{-L_{n}}=:\left(\left(w_{i j}\right)\right)
$$

is the triangle matrix with $w_{i j}=0$ if $1 \leq j<i \leq n$ and $w_{j j}=\mu_{j}$ if $1 \leq j \leq n$. This implies that the eigenvalues of $M_{n}$ are listed on the main diagonal of the product, as desired. The implementation has not been so evident.

At first, we recall two identities from [15]. 
Lemma 1. Let $M, m \in \mathbf{N}_{0}$ and $a, b \in \mathbf{R}$. Then

$$
\sum_{k=0}^{M}\left(\begin{array}{c}
a+k \\
k
\end{array}\right)\left(\begin{array}{c}
b-k \\
M-k
\end{array}\right)=\sum_{k=0}^{M}\left(\begin{array}{c}
a+b-k \\
M-k
\end{array}\right)
$$

and

$$
\sum_{k=0}^{M}(-1)^{k}\left(\begin{array}{c}
M \\
k
\end{array}\right)\left(\begin{array}{c}
a-k \\
m
\end{array}\right)=\left(\begin{array}{c}
a-M \\
m-M
\end{array}\right) .
$$

Proof. See formulas (43) on page 618 and (56) on page 619 of [15].

Let us introduce the matrix $L_{n}(\theta)=\left(\left(l_{i j}\right)\right)$ with the entries $l_{i j}=0$ for all $1 \leq i, j \leq n$ but for $i=j+1$, where

$$
l_{j+1, j}=-\left(\frac{(j+1) j \Theta(n-j-1)}{\Theta(n-j)}\right)^{1 / 2}, \quad 1 \leq j \leq n-1 .
$$

Consider the powers $L_{n}^{k}(\theta)=:\left(\left(l_{i j}^{(k)}\right)\right), 0 \leq k \leq n-1$. The nonzero entries of $L_{n}^{k}(\theta)$ fill up the $k$ th, $1 \leq k \leq n-1$, diagonal under the main one. By induction, we observe that

$$
\begin{aligned}
l_{j+k, j}^{(k)} & =l_{j+k, j+1}^{(k-1)} l_{j+1, j} \\
& =l_{j+k, j+k-1} l_{j+k-1, j+k-2} \cdots l_{j+1, j} \\
& =(-1)^{k} \prod_{r=0}^{k-1}((j+r+1)(j+r))^{1 / 2}\left(\prod_{r=0}^{k-1} \frac{\Theta(n-j-r-1)}{\Theta(n-j-r)}\right)^{1 / 2} \\
& =(-1)^{k}(j)_{k}\left(\frac{j+k}{j}\right)^{1 / 2}\left(\frac{\Theta(n-j-k)}{\Theta(n-j)}\right)^{1 / 2}
\end{aligned}
$$

if $1 \leq k \leq n-j$. Hence the matrix $V:=\mathrm{e}^{L_{n}(\theta)}=:\left(\left(v_{i j}\right)\right)$ has $v_{i j}=0$ if $1 \leq i<j \leq n$ and

$$
\begin{aligned}
v_{i j} & =\frac{l_{i j}^{(i-j)}}{(i-j) !}=(-1)^{i-j}\left(\begin{array}{c}
i-1 \\
j-1
\end{array}\right)\left(\frac{i}{j}\right)^{1 / 2}\left(\frac{\Theta(n-i)}{\Theta(n-j)}\right)^{1 / 2} \\
& =(-1)^{i-j}\left(\begin{array}{l}
i \\
j
\end{array}\right)\left(\frac{j}{i}\right)^{1 / 2}\left(\frac{\Theta(n-i)}{\Theta(n-j)}\right)^{1 / 2}
\end{aligned}
$$

if $i \geq j$. Moreover, $V^{-1}=\mathrm{e}^{-L_{n}(\theta)}=\left(\left(\left|v_{i j}\right|\right)\right)$ if $1 \leq i, j \leq n$. 
More technical obstacles arise calculating

$$
\begin{aligned}
w_{i j}= & \sum_{\substack{1 \leq r \leq i \\
j \leq s \leq n}} v_{i r} m_{r s}\left|v_{s j}\right| \\
= & \sum_{\substack{1 \leq r \leq i \\
j \leq s \leq n}}(-1)^{i-r}\left(\begin{array}{c}
i \\
r
\end{array}\right)\left(\frac{r}{i}\right)^{1 / 2}\left(\frac{\Theta(n-i)}{\Theta(n-r)}\right)^{1 / 2} \cdot \frac{\Theta(n-r-s)}{(r s \Theta(n-r) \Theta(n-s))^{1 / 2}} \\
& \times\left(\begin{array}{c}
s-1 \\
j-1
\end{array}\right)\left(\frac{s}{j}\right)^{1 / 2}\left(\frac{\Theta(n-s)}{\Theta(n-j)}\right)^{1 / 2} \\
& -\sum_{\substack{1 \leq r \leq i \\
j \leq s \leq n}}(-1)^{i-r}\left(\begin{array}{c}
i \\
r
\end{array}\right)\left(\frac{r}{i}\right)^{1 / 2}\left(\frac{\Theta(n-i)}{\Theta(n-r)}\right)^{1 / 2} \cdot\left(\frac{\Theta(n-r)}{r \Theta(n)}\right)^{1 / 2}\left(\frac{\Theta(n-s)}{s \Theta(n)}\right)^{1 / 2} \\
& \times\left(\begin{array}{c}
s-1 \\
j-1
\end{array}\right)\left(\frac{s}{j}\right)^{1 / 2}\left(\frac{\Theta(n-s)}{\Theta(n-j)}\right)^{1 / 2} \\
= & : \Sigma_{1}-\Sigma_{2} .
\end{aligned}
$$

Here

$$
\Sigma_{1}=(-1)^{i}\left(\frac{\Theta(n-i)}{i j \Theta(n-j)}\right)^{1 / 2} \sum_{1 \leq r \leq i \wedge n-j} \frac{(-1)^{r}}{\Theta(n-r)}\left(\begin{array}{c}
i \\
r
\end{array}\right) \sum_{j \leq s \leq n-r} \Theta(n-r-s)\left(\begin{array}{l}
s-1 \\
j-1
\end{array}\right) .
$$

After the change $s=n-r-k$, the inner sum reduces to that given in (10). So we obtain

$$
\begin{aligned}
& \sum_{k=0}^{n-r-j}\left(\begin{array}{c}
\theta-1+k \\
k
\end{array}\right)\left(\begin{array}{c}
n-r-1-k \\
n-r-j-k
\end{array}\right)=\sum_{k=0}^{n-r-j}\left(\begin{array}{c}
\theta+n-r-2-k \\
n-r-j-k
\end{array}\right) \\
& =\sum_{l=0}^{n-r-j}\left(\begin{array}{c}
\theta-2+j+l \\
l
\end{array}\right)=\left[x^{n-r-j}\right] \frac{1}{(1-x)^{\theta+j}}=\left(\begin{array}{c}
\theta+n-r-1 \\
n-r-j
\end{array}\right) .
\end{aligned}
$$

Hence

$$
\Sigma_{1}=(-1)^{i}\left(\frac{\Theta(n-i)}{i j \Theta(n-j)}\right)^{1 / 2} \sum_{1 \leq r \leq i \wedge n-j} \frac{(-1)^{r}}{\Theta(n-r)}\left(\begin{array}{c}
i \\
r
\end{array}\right)\left(\begin{array}{c}
\theta+n-r-1 \\
n-r-j
\end{array}\right) .
$$

Similarly,

$$
\Sigma_{2}=\frac{(-1)^{i}}{\Theta(n)}\left(\frac{\Theta(n-i)}{i j \Theta(n-j)}\right)^{1 / 2} \sum_{1 \leq r \leq i}(-1)^{r}\left(\begin{array}{l}
i \\
r
\end{array}\right) \sum_{j \leq s \leq n} \Theta(n-s)\left(\begin{array}{c}
s-1 \\
j-1
\end{array}\right) .
$$


Since

$$
\sum_{j \leq s \leq n}\left(\begin{array}{c}
s-1 \\
j-1
\end{array}\right) \Theta(n-s)=\left[x^{n}\right]\left(\frac{x^{j}}{(1-x)^{j}} \cdot \frac{1}{(1-x)^{\theta}}\right)=\left(\begin{array}{c}
\theta+n-1 \\
n-j
\end{array}\right),
$$

we obtain

$$
\Sigma_{2}=\frac{(-1)^{i+1}}{\Theta(n)}\left(\frac{\Theta(n-i)}{i j \Theta(n-j)}\right)^{1 / 2}\left(\begin{array}{c}
\theta+n-1 \\
n-j
\end{array}\right) .
$$

Consequently,

$$
\begin{aligned}
w_{i j} & =\Sigma_{1}-\Sigma_{2} \\
& =(-1)^{i}\left(\frac{\Theta(n-i)}{i j \Theta(n-j)}\right)^{1 / 2} \sum_{0 \leq r \leq i \wedge n-j} \frac{(-1)^{r}}{\Theta(n-r)}\left(\begin{array}{c}
i \\
r
\end{array}\right)\left(\begin{array}{c}
\theta+n-r-1 \\
n-r-j
\end{array}\right) \\
& =:(-1)^{i}\left(\frac{\Theta(n-i)}{i j \Theta(n-j)}\right)^{1 / 2} \cdot \Sigma .
\end{aligned}
$$

Using the definition of $\Theta(m)$ and applying identity (11), we find that

$$
\Sigma=\frac{j !}{(\theta)_{j}} \sum_{0 \leq r \leq i \wedge n-j}(-1)^{r}\left(\begin{array}{c}
i \\
r
\end{array}\right)\left(\begin{array}{c}
n-r \\
j
\end{array}\right)=\frac{j !}{(\theta)_{j}}\left(\begin{array}{c}
n-j \\
j-i
\end{array}\right) .
$$

Here $\Sigma=0$ if $j<i$ and $\Sigma=j ! /(\theta)_{j}$ if $i=j$. Plugging this into (12), we obtain

$w_{i j}=(-1)^{i}\left(\frac{\Theta(n-i)}{i j \Theta(n-j)}\right)^{1 / 2} \frac{j !}{(\theta)_{j}}\left(\begin{array}{c}n-j \\ j-i\end{array}\right)= \begin{cases}0 & \text { if } i>j \\ (-1)^{j}(j-1) ! /(\theta)_{j} & \text { if } i=j .\end{cases}$

This proves the main assertion of Theorem 2 .

It remains to find

$$
q_{1}(j)=[(\theta+2) j-(2 n+\theta)] / 2(1-n), \quad 1 \leq j \leq n,
$$

define $\bar{e}_{2}$, and show that $\bar{e}_{2} M_{n}=\mu_{2} \bar{e}_{2}$. Since the latter is the subject of Theorem 3, we may omit the proof.

Theorem 2 is proved.

Remark. The following observation is worth mentioning. As it stems from the proof, $w_{n i}=w_{i n}=\mu_{n} \delta_{i n}$ if $1 \leq i \leq n$, where $\delta_{i n}$ is the Kronecker symbol. Hence the vector $\bar{v}_{n}$ is the eigenvector of $M_{n}$ corresponding to $\mu_{n}$. 
Thus, it is proportional to the vector $\bar{e}_{n}$ from the next theorem. To verify the mentioned property for $\bar{e}_{n}$, it suffice to apply a formula established even before the Gauss' seminal paper from 1812 on the hypergeometric series (see [2, Lecture 7] for the historical account). The $\mathrm{Chu-Vandermonde} \mathrm{formula}$ ((7.16), p. 59 of the same book) reads as follows:

$$
{ }_{2} F_{1}(-m, b ; c ; 1)=\frac{(c-b)_{m}}{(c)_{m}} .
$$

Hence

$q_{n-1}(j)={ }_{2} F_{1}(-j+1, \theta+n ; 2 ; 1)=\frac{(2-n-\theta)_{j-1}}{(2)_{j-1}}=\frac{(-1)^{j-1}(\theta+n-j)_{j-1}}{j !}$.

This in turn yields that $\bar{e}_{n} M_{n}=\mu_{n} \bar{e}_{n}$.

\section{Proof of Theorem 3}

We now find all eigenvectors of the matrix $M_{n}$. Again, we have to recall a useful identity.

Lemma 2. Let $p, q, M \in \mathbf{N}_{0}, \alpha, \beta \in \mathbf{R}$, and $a_{1}, \ldots, a_{p} ; b_{1} \ldots, b_{q}$ be the $p a$ rameters such that the hypergeometric series below is correctly defined. Then

$$
\begin{aligned}
& \sum_{k=0}^{M}\left(\begin{array}{c}
M \\
k
\end{array}\right)(\alpha)_{M-k}(\beta)_{k} \cdot{ }_{p+1} F_{q}\left(-k,\left(a_{p}\right) ;\left(b_{q}\right) ; 1\right) \\
& =(\alpha+\beta)_{M} \cdot{ }_{p+2} F_{q+1}\left(-M, \beta,\left(a_{p}\right) ; \alpha+\beta,\left(b_{q}\right) ; 1\right) .
\end{aligned}
$$

Proof. See formula (7) presented on page 388 in [16].

As a corollary, we find the next sum.

Lemma 3. Let $n \geq 2,0 \leq M \leq n-1$ and $0 \leq r \leq n-1$. Then

$$
\begin{aligned}
\Sigma_{r}(M) & :=\sum_{k=0}^{M} Q_{r}(k ; 1, \theta-1, n) \Theta(M-k) \\
& =\frac{(\theta+1)_{M}}{M !}{ }_{4} F_{3}(-M, 1,-r, r+\theta+1 ; \theta+1,2,1-n ; 1) .
\end{aligned}
$$


Proof. Apply Lemma 2 for $\alpha=\theta, \beta=1$, and $p=q=2$.

The obtained expressions of $\Sigma_{r-1}(M), 1 \leq r \leq n$ will be used afterwards. For short, let

$$
{ }_{4} F_{3}(-M)={ }_{4} F_{3}(-M, 1,1-r, r+\theta ; \theta+1,2,1-n ; 1) .
$$

Lemma 4. Let $\bar{y}_{r}=\left(y_{r 1}, \ldots, y_{r n}\right)=\pi_{r-1} \bar{e}_{r} M_{n}, 1 \leq r \leq n$, then

$$
y_{r i}=-\left(\frac{\Theta(n-i)}{i}\right)^{1 / 2} \frac{n}{r(r+\theta-1)} \cdot{ }_{3} F_{2}(-r,-n+i, r+\theta-1 ; \theta,-n ; 1)
$$

if $1 \leq i \leq n$.

Proof. In the notation above,

$$
\begin{aligned}
y_{r i} & =\frac{1}{(i \Theta(n-i))^{1 / 2}} \Sigma_{r-1}(n-i-1)-\left(\frac{\Theta(n-i)}{i}\right)^{1 / 2} \frac{1}{\Theta(n)} \Sigma_{r-1}(n-1) \\
& =\frac{1}{\theta}\left(\frac{\Theta(n-i)}{i}\right)^{1 / 2}\left[(n-i) \cdot{ }_{4} F_{3}(-n+i+1)-n \cdot{ }_{4} F_{3}(-n+1)\right]
\end{aligned}
$$

if $1 \leq i<n$. Since $(a)_{l-1}=(a-1)_{l} /(a-1)$ if $a \neq 1$, we have

$$
\begin{aligned}
& (n-i) \cdot{ }_{4} F_{3}(-n+i+1)=(n-i) \sum_{l=1}^{r} \frac{(-n+i+1)_{l-1}(1-r)_{l-1}(r+\theta)_{l-1}}{(\theta+1)_{l-1}(-n+1)_{l-1} l !} \\
& =-\frac{\theta n}{r(r+\theta-1)}\left[-1+\sum_{l=0}^{r} \frac{\left.(-n+i)_{l}(-r)_{l}(r+\theta-1)_{l}\right]}{(\theta)_{l}(-n)_{l} l !}\right] \\
& =\frac{\theta n}{r(r+\theta-1)}\left[1-{ }_{3} F_{2}(-r,-n+i, r+\theta-1 ; \theta,-n ; 1)\right] .
\end{aligned}
$$

Similarly,

$$
\begin{aligned}
n \cdot{ }_{4} F_{3}(-n+1) & =\frac{\theta n}{r(r+\theta-1)}\left[1-{ }_{3} F_{2}(-r,-n, r+\theta-1 ; \theta,-n ; 1)\right] \\
& =\frac{\theta n}{r(r+\theta-1)}\left[1-{ }_{2} F_{1}(-r, r+\theta-1 ; \theta ; 1)\right] \\
& =\frac{\theta n}{r(r+\theta-1)},
\end{aligned}
$$


by virtue of $(\underline{13})$. Hence

$$
\begin{aligned}
& (n-i) \cdot{ }_{4} F_{3}(-n+i+1)-n \cdot{ }_{4} F_{3}(-n+1) \\
& =-\frac{\theta n}{r(r+\theta-1)} \cdot{ }_{3} F_{2}(-r,-n+i, r+\theta-1 ; \theta,-n ; 1) .
\end{aligned}
$$

Plugging this into the previous expression of $y_{r i}$, we complete the proof in the case $i<n$.

If $i=n$, then, using Lemma 3 and (15), we obtain

$$
\begin{aligned}
y_{r n} & =-\frac{1}{\sqrt{n} \Theta(n)} \Sigma_{r-1}(n-1)=-\frac{1}{\sqrt{n} \Theta(n)} \cdot \frac{(\theta+1)_{n-1}}{(n-1) !} \cdot \frac{\theta}{r(r+\theta-1)} \\
& =-\frac{\sqrt{n}}{r(r+\theta-1)} .
\end{aligned}
$$

This is consistent with expression (14) given in the lemma.

Lemma 4 is proved.

Proof of Theorem 3. Let $1 \leq r \leq n$ be fixed. Recall that $q_{k}(x), 0 \leq k \leq r$, span the subspace of polynomials whose degrees do not exceed $r$. Analyse the polynomial appearing in Lemma 4, namely,

$$
\Phi_{r}(x)={ }_{3} F_{2}(-r,-n+x, r+\theta-1 ; \theta,-n ; 1) .
$$

As we have seen proving (15), we have $\Phi_{r}(0)=0$. Hence $x^{-1} \Phi_{r}(x)$ is a polynomial of degree $r-1$ and

$$
\Phi_{r}(x)=x \sum_{k=0}^{r-1} c_{k} q_{r}(x), \quad c_{k} \in \mathbf{R} .
$$

The leading coefficients of the polynomials $\Phi_{r}(x)$ and $q_{r}(x)$ are, respectively,

$$
\frac{(-1)^{r}(r+\theta-1)_{r}}{(\theta)_{r}(-n)_{r}}, \quad \frac{(r+\theta)_{r-1}}{r !(-n+1)_{r-1}}
$$

Consequently,

$$
c_{r-1}=\frac{(-1)^{r}(r+\theta-1)_{r}}{(\theta)_{r}(-n)_{r}} \cdot \frac{r !(-n+1)_{r-1}}{(r+\theta)_{r-1}}=\frac{(-1)^{r-1} r !(r+\theta-1)}{(\theta)_{r} n} .
$$


Now, the result of Lemma 4 can be rewritten as follows:

$$
\begin{aligned}
y_{r i} & =-\left(\frac{\Theta(n-i)}{i}\right)^{1 / 2} \frac{n i}{r(r+\theta-1)}\left[c_{r-1} q_{r-1}(i)+\sum_{k=0}^{r-2} c_{k} q_{k}(i)\right] \\
& =(i \Theta(n-i))^{1 / 2}\left[\frac{(-1)^{r}(r-1) !}{(\theta)_{r}} q_{r-1}(i)+\sum_{k=0}^{r-2} d_{k} q_{k}(i)\right]
\end{aligned}
$$

with some coefficients $d_{k}=d_{k}(n, r, \theta)$ for each $1 \leq i, r \leq n$. Note that the fraction in the brackets is just $\mu_{r}$ found in Theorem 2 .

For $1 \leq r \leq l \leq n$, applying the last formula and the definition of the inner product (9), we obtain

$$
\begin{aligned}
\bar{e}_{l} M_{n} \bar{e}_{r}^{\prime} & =\pi_{r-1}^{-1} \bar{e}_{l} y_{r}^{\prime}=\frac{1}{\pi_{l-1} \pi_{r-1}}\left[\mu_{r}<q_{l-1}, q_{r-1}>+\sum_{k=0}^{r-2} d_{k}<q_{l-1}, q_{k}>\right] \\
& =\mu_{r} \delta_{r l}
\end{aligned}
$$

by virtue of orthogonality. This shows that each $\bar{e}_{r}$ in the basis is the eigenvector for $M_{n}$ corresponding to $\mu_{r}$.

Theorem 3 is proved.

Concluding remark. Comparing the expression $\bar{e}_{r} M_{n}=\mu_{r} \bar{e}_{r}$ with $\pi_{r-1}^{-1} \bar{y}_{r}$ given by Lemma 4, we arrive to a seemingly new relation of the generalized hypergeometric functions. For $1 \leq i, r \leq n$ and $\theta>0$, it holds that

$$
\begin{aligned}
(-1)^{r-1} r ! i \cdot{ }_{3} F_{2}(-r+1, & -i+1, r+\theta ; 2,-n+1 ; 1) \\
& =(\theta)_{r-1} n \cdot{ }_{3} F_{2}(-r,-n+i, r+\theta-1 ; \theta,-n ; 1) .
\end{aligned}
$$

Derivation of it using an appropriate sequence of the so-called contiguous relations (see [17]) would not be short.

\section{References}

[1] Arratia, R., Barbour, A. D., and Tavaré, S. (2003) Logarithmic Combinatorial Structures: a Probabilistic Approach. EMS Monographs in Mathematics, EMS Publishing House, Zürich. 
[2] Askey, R. (1975) Polynomials and Special Functions. Soc. Industr. and Applied Maths, Philadelphia, Pensylvania.

[3] Beals, R. and Wong, R. (2012) Special Functions. Cambridge University Press, Cambridge.

[4] Crane, H. (2016) The ubiquitous Ewens sampling formula. Statist. Sci. 31 1-19.

[5] Ewens, W. J. (1972) The sampling theory of selectively neutral alleles. Theor. Pop. Biol. 3 87-112.

[6] Feng, Sh. (2010) The Poisson-Dirichlet Distribution and Related Topics. Springer, Berlin.

[7] Johnson, N. S., Kotz, S., and Balakrishnan, N. (1997) Discrete Multivariate Distributions. Wiley, New York.

[8] Klimavičius, J., and Manstavičius, E. (2018) The Turán-Kubilius inequality on permutations. Annales Univ. Sci. Budapest., Sect. Comp. 48 45-51.

[9] Kubilius, J. (1983) Estimating the second central moment for strongly additive arithmetic functions (Russian). Liet. matem. rink., 23 110-117; translation in Lith. Math. J. 23 61-69.

[10] Manstavičius, E. (2006) Conditional Probabilities in Combinatorics. The Cost of Dependence. In: Prague Stochastics. 7th Prague symposium on asymptotic statistics and 15th Prague conference on information theory, statistical decision functions and random processes (M. Huškov'a and M. Janžura, eds), Prague, Charles University, Matfyzpress, 523-532.

[11] Manstavičius, E. (2007) Moments of additive functions defined on the symmetric group, Acta Appl. Math. 97 119-127.

[12] Manstavičius, E. (2009) An analytic method in probabilistic combinatorics. Osaka J. Math. 46 273-290.

[13] Manstavičius, E., and Stepanauskas, V. (2014) On variance of an additive function with respect to a generalized Ewens probability. In: Proceedings of the 25th International Conference on Probabilistic, Combinatorial and Asymptotic Methods for the Analysis of Algorithms, Discrete Math. 
Theor. Comput. Sci. Proc., BA, Assoc. Discrete Math. Theor. Comput. Sci., Nancy, 301-311.

[14] Manstavičius, E., and Žilinskas, Ž. (2011) On a variance related to the Ewens sampling formula. Nonlinear Anal. Model. Control 16 453-466.

[15] Prudnikov, A. P., Brychkov, Yu. A., and Marichev, O. I. (1998) Integrals and Series, vol. 1. Taylor \& Francis, Fourth printing.

[16] Prudnikov, A. P., Brychkov, Yu. A., and Marichev, O. I. (1990) Integrals and Series, vol. 3, More Special Functions. Gordon and Breach Sci. Publ., New York.

[17] Rainville, E. D. (1960) Special Functions. Macmillan, New York.

[18] Watterson, G. A. (1974) The sampling theory of selectively neutral alleles diffusion model. Advances in Appl. Probab., 6 463-488.

[19] Wieand, K. (1998) Eigenvalue Distributions of Random Matrices in the Permutation Group and Compact Lie Groups. PhD thesis, Harvard University.

[20] Zacharovas, V. (2004) Distribution of the logarithm of the order of a random permutation (Russian). Liet. mat. rink. 44 372-406; translation in Lithuanian Math. J. 44 296-327.

Affiliation of the authors: Institute of Mathematics, Vilnius University, Naugarduko str. 24, LT-03225 Vilnius, Lithuania Corresponding author: Eugenijus Manstavičius, email: eugenijus.manstavicius@mif.vu.lt 